\title{
Live Streaming Platforms and the Critical Discourse About Video Games
}

\section{Research goals and methodology}

The aim of this article is to assess the potential and importance of online streaming services that focus on game content for the critical discourse about games. To do this, my research will approach this issue from two different angles. The first will present an overview of the methods that can potentially help in researching broadcasters and fans' activity as it pertains to the critique of video games. It will be done according to a three-part paratextual framework based on the work of Mia Consalvo (2007), to be elaborated in the "Paratextual framework for game-centered streaming services" section of this work. The second will be focused on Veli-Matti Karhulahti's (2016) concepts of interview frame and play frame, derived from Erving Goffman's frame analysis, which will be used to analyze the two distinctive modes of interaction with online streaming media. My final section will present examples of how players' practices, as well as strategies employed by streamed services themselves, are used to shape the current discourse of and about video games. The main research question concerns the potential of streaming services to facilitate critical discourse about games. By "discourse", I refer to a "primarily instrumental device that can foster common perceptions and understandings for specific purposes" (Howarth, 2000, p. 3). This definition allows me to bring together the data from various approaches to studies of streaming platforms and confront them with my own research on Twitch.tv and Douyu.com, concerning these platforms' influence on shaping the opinions, perceptions and journalist-like criticism about video games. 
The interview frame deals with the conversation practices of the Twitch.tv chat, while the play frame refers to the competitive aspects of the broadcasted gameplay. I am particularly interested in the actions that disturb these kinds of frames, but remain a part of the communication process that eventually increases the attractiveness of Twitch.tv as a medium. Karhulahti uses the well-known concepts of Erwin Goffman to discern two simultaneously occurring visual and textual events - the act of player/audience interaction and the act of player/game interaction. He focuses on specific broadcasting tactics that involve both streamers' and their audience's disruptive behaviour, which may at times interfere with any of the play frames. These elements are included to connect the paratextual framework with a closer look at particular practices and tactics involved in two streaming platforms: Twitch.tv and Chinese-focused Douyu.com.

Various theories of paratexts have already been used in game studies to analyze phenomena like game trailers (Švelch, 2015) or the theorycrafting practices in video game fandoms (Paul 2011). In the analysis of the critical discourses which stem from the usage of streaming media services, I decided to base my research on the work of Mia Consalvo and her framework dividing the influence of paratexts into three fields: ga meplay, players, and gaming capital. In her book about cheating she treats gaming magazines, discussion boards, podcasts and other sources of gamers' knowledge outside the games themselves as paratextual industries, writing that "paratextual industries (...) work to define what they believe is acceptable gameplay as well as the right type of player with the correct form of gaming capital" (Consalvo, 2007, p. 147). I would say that this category really fits the streaming services as they are in the current media landscape

In this paper, I will begin with briefly characterizing Twitch.tv and Douyu.com as distinct examples of game-focused streaming services (section 1). Then, I will move on to the topic of game criticism, and how the work of streamers as content creators can influence the reception of video games (sections 2 and 3). Afterwards, I will propose a reading of analyzed phenomena based on paratextual framework (section 4). Lastly, I will summarize my findings with the perspective of changing viewership figures in relation to selected ludic properties of games (section 5).

\section{Twitch.tv and Douyu.com as streaming media}

In this article, I will take the perspective of an individual streamer rather than a corporate entity, regardless of the dominant position of the branded and promotional content of the latter in both analyzed services. Case studies presented in the subsequent sections are extracted from data based on one year of participatory observation, conducted on a group of streamers with at least 1000 subscribers. Both Twitch.tv and 
Douyu.com are leaders in the category of Internet streaming platforms, with over 10 and 1.6 million of unique page views respectively ${ }^{1}$. While analysis of streaming services, whether connected to video gaming or not, is a relatively new topic for scientific research (see Gros et al. 2017), their growing importance from an economic standpoint has created the need for in-depth inquiries.

Discussing the question of Twitch.tv as a platform for video game criticism inevitably brings up the issue of control: who decides if a particular material is suitable for criticism, or which parts of the gameplay can be publicly shown. Furthermore, there are cases of developers who enforced bans on streamers who broadcasted footage from inappropriate versions of the game or even those who published gameplay material deemed by the company as "not fun to watch" (see Jungar, 2016, p. 26). Game developers - even those not particularly interested in creating products which fit streaming services' audience-broadcaster business model - admit that "we live in a world now in which watching other people play video games, whether via Twitch or video streaming, is now key" (Fargo, 2017). Online streaming services are places of equal interest for players, developers and content creators alike (see Gandolfi, 2016). Streaming services have overtaken other media when it comes to video game footage: "videogame enthusiasts that previously put videos of gameplay up on YouTube (Let's Plays) are now either also on or transitioning entirely to Twitch" (Consalvo, 2017, p. 85). Comparing the two main sources of paratextual streaming video content in different cultural contexts - predominantly English-speaking Twitch.tv and Mandarin-focused Douyu.com - allows us to confront and compare different models, around which discourse in and on video games is currently shaped.

Chinese streaming platform Douyu.com strongly resembles Twitch.tv on the interface level: similarly placed sections are dedicated to the streamers' cameras; chat, by default, occupies comparable screen size; the layout of icons and technical data is comparable to the Amazon-owned service's GUI. Yet significant differences are noticeable when looking at the interview frame. There are more buttons responsible for engaging with the stream by supporting the broadcaster financially and/or demanding the live appearance of particular content on Douyu.com. This feature can be partially attributed to the different model of engagement for Chinese video game enthusiasts who are willing to participate in live streams; as confirmed by the research study done by Lu et al. (2017), "reward-based systems and fan groups on instant messaging apps further afford social interactions between viewers and streamers and facilitate community

1 Data according to hypestat.com. 
building". Live streaming in China remains an under-researched topic, but even now the preliminary survey results suggest the prevalence of the varied streamer category, even in the case of professional e-sport streamers. This may indicate the correlation between the streamer's popularity and credibility, not only as a specialist in the niche activity of playing video games on expert level, but in general as a person whose cultural taste is something worth following. The fragment of one of the interviews cited by Lu et al. (2017) quotes the ability to sing and having "good music taste" among the qualities of an esteemed streamer. These often undervalued "cultural/all-round credibility" categories remain an important factor for building individual credibility for a streamer: they can effectively becomes a figure whose particular gameplay preferences can dictate short-term in-game economy. Such is the case of popular Path of Exile streamer Kostya Khudoshin (better known as Mathil), whose ability to influence global prices for particular in-game items just by streaming gameplay footage of a particular character he's using has been nicknamed the "Mathil effect" by the community of fans, and became a running joke on Reddit, YouTube and official game's forums $s^{2}$. Streamers can also build a special "aura" around their video game activities. Mathil regularly engages in physical training (other examples of such PoE oriented streamers include LiftingNerdBro ${ }^{3}$ ), while others might succeed in building their "reliable" persona outside of the gameplay. These "auras" are able to heavily influence critical discourse on a particular title. In a similar manner, streams which feature in-depth analysis and critique of patch notes form another category of game criticism, one which not only influences the ways in which the game is subsequently engaged with by the players (by promoting particularly effective strategies and signaling the advent of new possible tactics), but also how it is perceived by the developers, who must pay very close attention to the opinions of the influential streamers and make amendments based on their demands or critique in subsequent patches.

\section{Online streaming services as platforms for game criticism}

I would argue that with the advent of streaming services we witnessed an alternation (or to be more precise, remediation) of a special space connected with video game play, one similar to the private, corporeal-marked private space of the gamer described by Jesper Juul in A Casual Revolution (2009). It is a space occupied by a human body sitting in front of a computer, fragmented by the frame of reference provided by the web-

2 For details and data extracted from trading Path of Exile sites confirming the validity of „Mathil effect", see https://www.reddit.com/r/pathofexile/comments/7ta604/mathil_effect_at_its_finest/

3 For reference, see https://www.twitch.tv/lifting 
cam. These are the small screens with hands on the keyboard or gamepad, Skype-alike movie mugshots of streamers, or even multiple screens showing simultaneous gaming (and non-gaming) footage just as the CCTV office cameras, such as in the Streamers's House project ${ }^{4}$ The corporeal dimension of streams has been critically acknowledged by Sky LaRell Anderson (2017) in her study on Twitch.tv. Aderson's approach relied on implementing Grounded Theory to study various forms of "interactive online corporeality" of streamers, focusing mainly on the strategies used by the website.

Among key features of Twitch.tv and similar streaming platforms, few can be acknowledged as crucial in distinguishing this kind of service from the other popular cyber media dealing with video game-based content. I would like to focus on just two of them: the potential to turn a single-player experience into a social activity (Consalvo, 2017) and the ability to create and maintain long-term commitments attributed to particular channels as specific "places" for the community (Hamilton et al. 2014). Both involve looking into the status of Twitch.tv and Douyu.com content creators, as well as assessing the fan-made paratextual framework they operate in.

\section{Content creators / game critics}

The first question regarding the discursive power of streaming services is the status of their content. Most popular streamers, as previously mentioned, also serve as game critics, yet their message hardly ever meets journalist standards of objective critique. In a way, when someone from the audience directly asks a streamer for an opinion, they can already formulate the answer based on streamer's behavior in the game or his general attitude towards a given product, which can hardly be masked during long, unedited play sessions. The discussion about what constitutes video game criticism and what does not is undeniably important, but due to the relatively non-hierarchical circulation of content it may be less crucial than in the other, older forms of digital and non-digital media. Given the current trends in game studies research, further investigation on this topic may be troublesome, as it would include cross-disciplinary comparisons conducted that must account for theories that are deeply grounded in existing idiosyncrasies of long-established disciplines (see Deterding, 2017). One of the aims of this article is to assess the importance of finding the main circulation of critical discourse of video games on digital streaming platforms. While by no means "egalitarian" or grassroot, they are nonetheless forced to acknowledge the players' tastes and are actively curated by the algorithms of viewership. For this analysis, I will stick to just one proposal regarding

4 http://streamerhouse.com/ 
the matter of video games criticism, mentioned in the panel discussion during 2009's DiGRA conference. At that time, Ian Bogost argued that individual uses or works done in a particular medium have a potential to critically resist or even alter the very properties of that medium. "[Criticism] treats individual works as important and meaningful, each one possessing its own properties that both combine with and resist those of the medium that encloses it." (Bogost, 2009) ${ }^{5}$.

This rather astute idea grasps the general modus operandi of streaming services in their current shape and scope - it is games that dictate the tools used for their criticism, and not so much the digital platform through which they are filtered while being streamed. Twitch.tv chat and YouTube streaming service are rather static, with regard to the interface and the policies of censorship they implement. The played and streamed product largely dictates the rules of interaction. If the game allows for some players (usually chosen from the channel's subscriber's list) to engage in online play with the streamer, this fact alone drastically changes the discursive frame of the streamed act of play. It is no coincidence that some of the most popular streamers, who are capable of building a sturdy group of players supporting them in their online gaming endeavors over time, experience a radical shift from "casual" gaming session to applied, broadcasted powerplay. Few resist temptations to capitalize on their follower's online work, refusing ingame goods or services offered to them for free (or just for a few seconds of online fame, when their avatar's name is recognized by the audience) by the devoted fans. Interestingly, there is evidence that such support can be met with accusations of cheating, especially if a particular streamer is capitalizing on digital goods earned by the fans during official race events or other types of online competitions ${ }^{6}$.

I would say that in the current state of Twitch.tv we witness something similar in the ways that streamers are using the medium constantly and critically approaching the bor-

5 Bogost, I. (2009). You Played That? Game Studies Meets Game Criticism. Panel Discussion Paper, DiGRA 2009. An example is the controversial "transfers" of in-game currency to the popular Path of Exile streamer Zizaran just after his character died in hardcore league (meaning he had to start over and quickly regain experience points to compete with the other players. He also is famous for his extremely long streaming sessions, which make his content available for the viewers in different time zones, thus helping him to gain recognition. For further fans' discussion on this topic, see https://www.reddit. com/r/pathofexile/comments/6gsj1b/ethical_handouts/ [accessed: 17.12.2016].

6 An example is the controversial "transfers" of in-game currency to the popular Path of Exile streamer Zizaran just after his character died in hardcore league (meaning he had to start over and quickly regain experience points to compete with the other players. He also is famous for his extremely long streaming sessions, which make his content available for the viewers in different time zones, thus helping him to gain recognition. For further fans' discussion on this topic, see https://www.reddit.com/r/ pathofexile/comments/6gsj1b/ethical_handouts/ [accessed: 17.12.2016]. 
ders in which it operates. An example of this is the ability viewers are afforded to make clips (short video excerpts from the stream, much alike the gifs). This feature was implemented to get rid of the need for using third-party sites outside Twitch to make and share such content. There is also an increasing awareness among the streamers concerning the viral potential of the clips. Some of the Twitch.tv content creators go as far as asking the audience to 'clip' selected events, with the intention to share the particularly interesting fragments of the broadcasts to the audiences absent during the live transmission.

\section{Paratextual framework for game-centered streaming services}

My claim is that Twitch.tv and Douyu.com have overtaken many of the functions that the paratextual industries from the pre-internet era had. Therefore, I will work with three concepts that relate to the persuasive work of these paratextual industries in the context of modern streaming services, which are parallel to the Consalvo's work on the culture of cheating (2007): the concept of acceptable gameplay, the concept of the right type of player, and the concept of correct form of gaming capital. I will examine each of them and provide examples of the research tools that can be used while investigating these elements. I have already commented on the usage of the paratextual theories in analyzing digital video games, but it is important to see the paratexts as elements of a broader picture:

If we imagine the triumvirate of Text, Audience, and Industry as the Big Three of media practice, then paratexts fill the space between them, conditioning passages and trajectories that criss-cross the mediascape, and variously negotiating or determining interactions among the three. (Gray, 2010, p. 23).

Twitch.tv is an example of a conglomerate of paratextual tools, and in the right context it might even be considered a paratext itself, as it influences the modern media landscape of video games more than any other digital platform (perhaps except for Valve's Steam). Twitch.tv is possessed by the video games' visual data, but at the same time it alters them in ways that expose their properties as interactive artifacts. It created a new type of video game worker - the figure of the streamer, whose impressive capability of popularizing a particular title is matched only by their high level of dependence on a particular audience. The major streaming services work as if they re-appropriated the user-generated content and proprietary video game software into an infinite media loop which feeds on the smaller circulations, spread ad hoc by its users. It is similar to the idea of a user-circulated web content (Jenkins, Ford, and 
Green, 2013, p. 15): while it still fits in the capitalist model of consumption and by itself is devoid of any subversive meaning, it nevertheless remains difficult to control or to be turned into a sellable commodity. This is the case of Twitch.tv clips: short video excerpts from streams which are usually spread, commented on and sometimes creatively altered in different types of Internet communication channels, with Reddit being one of the most popular as far as gaming culture is concerned.

The process of determining which elements appear on the visual interface of the analyzed services is governed by the algorithms, which take into consideration data such as viewership count and content flagging. This is usually done by streamers themselves as a form of declaration about which game they are currently streaming. Whereas this last strategy informs the potential viewers about the channel content, it is usually negligible in the case of most influential variety streamers; in such cases the actual streamed content would most likely remain inaccurately labeled by the algorithm, which operates with a slight delay in relation to the rapidly changing content of the stream.

\section{Acceptable gameplay}

Twitch.tv and other major games-centered streaming services are structured in a way that promotes the most popular games at any given time. On the front page, games are listed with the ones with the highest number of viewers at the top, with disregard to genres and brands. However, the front page also includes a window with a broadcast from a local streamer (relative to the viewer's location), who is currently promoted by the regional Twitch curator. These are usually region-locked, featuring channels from a language-sensitive roster, meaning that the viewers will likely be acquainted with the broadcasts in a language most fit to the IP address they log with. Such practice can already be considered the first threshold in determining acceptable gameplay - the model examples of it are visible as soon as the viewer enters the site, without the need to browse through the content.

This layout, present in Twitch.tv and similar services like Chinese Douyu.com, was contested by competing streaming services, although without much success. An example of this is smashcast.tv (rebranded after the merge of Azubu and Hitbox.tv), which is solely focused on e-sports and promoting models of broadcaster-audience communication methods alternative to the voice/chat paradigm. Among the advertised smashcast.tv's features the most prominent is a relatively low stream delay, which helps players engaged in games less represented on Twitch.tv, such as poker.

To study the gameplay mediated by these streaming services, one must place it in the context of digital tools that enable streamer-client communication. However, as was 
noticed by Daniel Recktenwald, "very few studies considered the language of gaming during actual instances of gaming". Crafting necessary tools to help investigating such time-sensitive process proved to be a difficult task. Recktenwald (2017a) proposes an approach that favors "discourse analytical tools to the transcripts of naturalistic online live stream data". In practice, he determined what the properties are of the spoken idiolect of streamers: how often they use particular words, whether they are spatial or time related, what parts of the screen they refer to when using the most common phrases. Afterwards, according to Recktenwald's method, this data must be compared with the data obtained from the written feedback of the chat. From this comparison, one can make claims about how streamers react to the spoken and written content provided by their viewers. Recktenwald's approach serves as a toolkit for analyzing discursive practices on Twitch.tv, which were already categorized within "cross-modal video-mediated communication" activities (Recktenwald, 2017b). It may well be used to find an answer to the question about Twitch's potential as a medium of game criticism. It can also help with more adequate assessment of the nature of both streamers' and audience work during the gaming session: how is a coherent discourse about a game formed, and is there any surplus value in the simultaneous dialogue between the gamer-broadcaster and their audience? The recent research $(\mathrm{Hu}$, Zhang, Wangon, 2017) into identification patterns and "continuance intention" of streamers and viewers alike shows that modern forms of watching other people play video games are instrumental in forming strong communities which spawn various models of consumption, which remain in the state of constant negotiation between the rules of the game, the particular streaming service, and both streamers and their audience. Assessing the factors which influence the audience response to the broadcasters' content may vary given the built-in mechanisms of gratification through monetary donations. Twitch.tv supports text messages attached to the donation notification, which may contain direct questions addressed to the broadcaster in hope to provoke the streamer to respond and disclose her/his views on a particular subject. Douyu.com conversely supports extensive system of "gifts" (Zhu et al. 2017), allowing the audience to engage in a specific "race" for attention of the streamer. Both of these methods bear heavy impact on gameplay, not only by disturbing the play frame, but also by shifting the focus towards the importance of paratextual production of the fans.

\section{The right type of player}

In the present state of digital video games entertainment sector, there are many instances that oversee the rules of interaction, from automated bot services which automatically exclude inappropriate words from the chat service, to the streamers them- 
selves occasionally banning viewers whose conduct they find offensive or simply not in congruence with the channel's proposed mode of entertainment. The category of the right player is bilateral: it pertains channel's viewers as well as a broadcaster. It can be argued that there is a set of factors which determine what type of the player is fit to successfully present a particular game. The argument cannot be reduced only to the genre or mechanics of the game, as among the top professional streamers there are many who are all-around entertainers, often switching from game to game and not committing to one particular title for prolonged periods of time A good example of one such streamer is content creator Lirik, who boasts the highest average viewers of the individual broadcasters on Twitch.tv, with numbers exceeding 25000 persons per broadcast ${ }^{7}$.

Streaming services could not maintain their influence on the players community were it not for the "right type of players": those who are critical enough to be believable, but at the same time present the act of play as an object of desire. Creating the desire in the mediated space of Twitch.tv and similar services can be viewed as a way of producing a special aura of commodity that the viewer can acquire and benefit from. However, there is still an important question of how to adequately measure the influence a streamer has on their community. The viewership number is one indicator, but the variety of interview frame types can vary significantly. While Alkaizer's (popular Path of Exile / Warcraft III streamer) chat is constantly spammed with ASCII images and redundant, mocking questions that remain unanswered, the communicator of DatModz (who is mainly a Diablo III streamer) is carefully moderated, and the interaction with his viewers remains at the center of the streamer's attention. It can be argued whether streaming services offer the potential to engage in critical play, and what type of play is even expected given the cultural context of the stream. In the Chinese streaming services, the various forms of streamer-audience interaction are rich and often taken for granted by the spectators, whose messages are often additionally displayed on the main gaming screen as a looped text (called danmu) - a visual strategy that is unheard of in any of the English-speaking services of this type. It obviously makes the gameplay content (the play frame) more difficult to follow but at the same tie it strengthens Karhulahti's (2016) interview frame, further emphasizing the importance of the mutual communication between the streamer and their audience.

7 Data retrieved from https://sullygnome.com/channels/365/watched, with research narrowed to data from the last 365 days. [Accessed: 28.08.2017]. 
Apart from the cultural context, one must be careful with the data obtained from the online tools used for quantifying Twitch.tv content. For example, the practice of participating in streamed play varies greatly, and may provide a very different user experience when conducted via phone, PC or a browser build into a gaming console. The practices connected with video game streaming content demand further attention and critical evaluation also in regard to the physical space they occur in, perhaps in the scope far exceeding that provided by Jesper Juul's A Casual Revolution (2009).

\section{The correct form of gaming capital}

Mia Consalvo claims that the concept of gaming capital "provides a key way to understand how individuals interact with games, information about games and the game industry, and other game players." (Consalvo, 2007, p. 4). It is useful in describing a dynamic environment of game fans, where knowledge often becomes a type of commodity - a gateway to get access to the "meta" strategies, a way of playing the game that is considered the most effective and thus "proper". I would argue that every gaming streamer faces a difficult problem: how to be relevant for their viewers in terms of both knowledge and gaming capital. While being an effective gamer and competent entertainer, i.e. finding balance between the play frame and interview frame, requires lot of effort, it's certainly the desired goal for some of the streamers ${ }^{8}$. The power play vs entertainment value equilibrium is equally troublesome to achieve in online video podcasts about video games, often hosted on Twitch.tv and other digital platforms. In other to attract the attention of viewers, the topics discussed must fit within the discourse presented in the other fans' paratextual industries. The risk of focusing solely on the issues that are important to the vocal minority of players (who happen to dominate in the various paratextual industries as discussed by Consalvo) is high, and significantly slows down the potential range and effectiveness of such fan-made incentives. Players invited by the gaming podcasts' hosts are usually doing unpaid, "promotional" work, while also advertising the game they invested large amounts of time and effort in, sharing their knowledge and potentially encouraging players to try a new

8 For a detailed discussion concerning the balance between delivering quality, long-time gameplay and gaining positive viewers' feedback because of other aspects of the channel, see https://www.reddit. com/r/pathofexile/comments/5inofd/the_ziz_effect/ (a fans' "case study" on Zizaran, one of the most popular Path of Exile streamers). 
product - or just sustain their interest by revealing different strategies for players to implement or new game goals to achieve.

Making use of the different forms of gaming capital in streamed media and finding tools to measure them effectively was one of the goals of the research conducted by Andreas Berglund et al. (2015). Their research used inductive analysis and interviews with streamers, finally proposing two models of streaming video game content: the game-centric model and the interaction model. The proposed thesis of said analysis also distinguishes between the "established" and "aspiring" streamers, differentiating between these two groups based on cross-media promotional strategies. In general, it was concluded that the interaction model suits the so-called "variety streamers", who in their broadcasting strategies prioritize interaction, then schedule, game choice, and lastly the visual aspects of the stream (Berglund et al., 2015, p. 29). Conversely, the game-centric model is represented by the "niched streamers", who prefer to focus on game choice first as important in achieving desired their effects, then broadcasting schedule, and lastly interaction and visual elements (p. 30). Interestingly, both groups mention visuals as the least crucial element of building a streamer's "brand", which may signify the strong position of a fixed and corporate-controlled interface of streaming services, which leaves little room for aesthetic innovation from the broadcasters aside from the simple graphic overlay for the main and pause screens. The research regrettably does not focus on the technical side of streaming, as it would be interesting to see how or whether the ability to properly set up a stream manifests as a form of gaming capital, and whether it influences the viewership and type of streamed content.

To sum up the part of the research focused on Mia Consalvo's approach to paratextual industries, one can conclude that acceptable gameplay, the right type of player and the correct form of gaming capital are all important factors in establishing and formatting the online video game streaming experience for both streamers and their viewers. While the concept of the author/audience dichotomy in the case of such media is not very useful, we can still assess the importance of streamers' work towards promoting a title among their viewing communities.

\section{The fragmented nature of streaming services}

Veli-Matti Karhulahti (2016) argues that the "three factors that are vital for the aesthetics of personal live streaming" relate to a feeling of affecting the stream, excitement derived from the suspense of waiting, and potential dramatic outcomes that develop during a live session. I would like to further explore these notions by 
acknowledging their potential as a means to express and share informed personal opinions about the games that are broadcasted during said individual streams.

The viewers' affection can be directed towards the streamer, the game, or (of course) both, although the research of Berglund et.al (2015) suggests that the dominant reaction is caused by the aforementioned three factors, according to the type of streamer. I would argue that "variety streamers" generate a slightly different type of paratextual "products of affection" (e.g. Twitch.tv clips, Reddit postings, memes or other visual material shared via Imgur or similar services) than "niched streamers". Whether paratexts produced in such a manner can be qualified as specimens of video game criticism is open for discussion, but their importance in creating Internet traffic that is important for PR reasons is hard to neglect.

Streaming services, as machines of paratextual dissemination, rely on creating the meaning and value constructed outside of the text - in this case a video game - but nevertheless still incorporate fragments into the framework of a new medium. The streamed data proliferates at a precipitous rate into numerous channels of communication - a Twitch.tv clip is being shared on Reddit or pasted into the other broadcasters' chat, to be commented on with related gif images based on the fans' gameplay footage. To actively participate in these dissipated instances of cultural associations means to embrace the gamers' idiolect and display a correct (e.g. readable by other users) form of gaming capital.

In a way, video streaming services capitalize on the promise of setting into motion the data that users are putting into them, which in principle makes them similar to computers. Commenting on the computers' capability to transfer and organize the data, Wendy Chun states their function as a "metaphor of the metaphor":

Computers, understood as universal machines, stand in for substitution itself. Allegedly making possible the transformation of anything into anything else via the medium of information, they are transference machines. They do not simply change $\mathrm{X}$ into $\mathrm{Y}$, they also animate both terms. They create a new dynamic reality: the files they offer us are more alive; the text that appears on their screens invites manipulation, addition, animation. (Chun, 2011, p. 57).

I would argue that similar processes of "creative substitution" are shaping the streaming media that relates to video games. Chat, the streamer's camera, the game's visual feedback, and algorithms governing the list of "recommended" streamers are all variables that demand defining from the end-user. If this happens, the viewer interacts 
with the broadcaster and sends a message; she writes herself in the chain of if-then scenarios, ready to be picked up by the other users, or even the code overseeing the actions of the Twitch bot. That being said, all of these meticulously crafted systems of data flow do not fully explain the life cycle of a game on a streaming service. To investigate the discursive underpinnings of this, we have to look into the machines that quantify the processing of the aforementioned data and juxtapose this with users' practices of interaction and play.

\section{Strategy games, viewership and popularity}

In 1993, an important event in the history of video game criticism occurred: Alan Emrich, an editor for Computer Gaming World, coined the term 4X, an abbreviation for eXplore, eXpand, eXploit, and eXterminate, naming a new video game subgenre. He was, at the same time, one of the designers of Master of Orion (1993, Simtex), a video game for which gameplay was so vividly summed up by the new genre name. The term itself was universally acclaimed by reviews when the game released, but the games designed according to the Emrich's manifesto are now a commercial margin of the video game industry. I would like to take this fact as an example of how strong Karhulahti's two last personal streaming categories - the need for suspense and anticipation of dramatic events - may be for the popularity and general volume of fans' paratexts produced for a given title.

The segment of high-budget strategy games belonging to the so-called $4 \mathrm{X}$ genre, although declining in popularity aside from a few strong franchises like Civilization, still provide powerful examples of ideological forces like historical determinism (Ghys, 2012) and postcolonial rhetoric (Ford, 2016), which underpin their mechanics and interaction patterns. While very interesting from a certain research angle, these titles provoke criticism and close reading mainly because of the inherent in-game narratives they impose onto players who wish to achieve success within their inner discursive framework. Player-to-player interactions are largely secondary to their overwhelmingly suggestive historical context, and the prevalence of turn-based combat in this type of product makes even the most graphically polished ones somewhat difficult (and perhaps simply boring) to follow if not engaged via direct playing experience. The following Figure 1 shows Sid Meier's Civilization VI average (green line) and peak (blue line) daily viewers, between Friday 23rd September 2016 and Monday 3rd July 2017. The game received significant viewers' attention for about three months after its launch, steadily declining without any significant peaks ever since. 


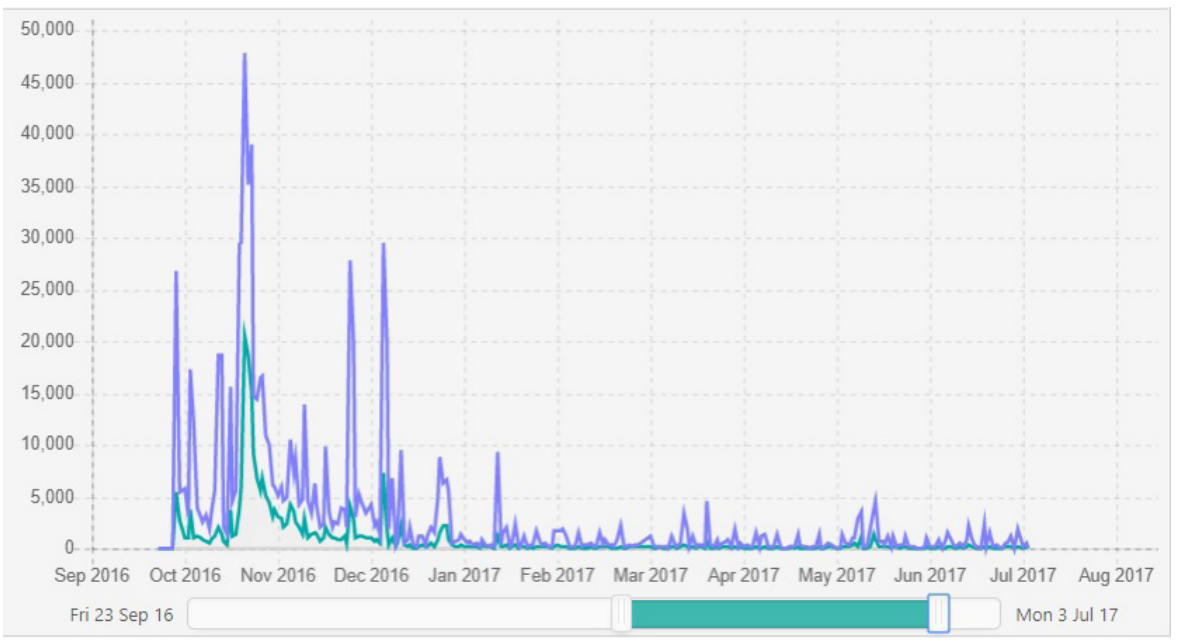

Figure 1. Sid Meier's Civilization VI average and peak daily viewers (SullyGnome.com, 20.08.2017) ${ }^{9}$

The high figures are compacted into short periods of time, during which the game appeared on the main Twitch.tv page and was simultaneously advertised as a major premiere in gaming media. Due to the nature of the gameplay (but, as we can conclude from Berglund's et al research, perhaps not the game's visuals), the audience's interest is not sustained by the new stimuluses, and streamers are not particularly interested in taking Civilization VI as their main streamed game. This last fact is perhaps the most telling: even the biggest franchises can be quickly capitalized by the big "variety" streamers, and when the media hype goes away, there is no one left to promote the game and introduce viewers to the more intricate features of a given production. In this aspect, the work of streamers and viewers is strictly interrelated: one group "feeds" on the interest of the second, and games are often reduced to the status of passable, easily consumable commodities. If they do not guarantee suspense, meaning their gameplay does not rely on dramatic change of events, or if there is no human factor (other players) involved in the gameplay process, the chance of dropping viewers rapidly after premiere is relatively high. The top 10 charts in the "most streamed games" ${ }^{10}$ category are constantly occupied almost exclusively by multiplayer titles with fast-paced gameplay, even if mechanically these games can be very repetitive or require long time investment from the players to achieve desired game goals.

9 https://sullygnome.com/game/Sid_Meiers_Civilization_VI

10 See https://sullygnome.com/games/365/streamed [accessed: 6.09.2017]. 


\section{Summary}

The two-fold aim of this article was to point out the possible research methods regarding online streaming services as mediums for video game criticism, and to assess the potential of these platforms for generating critical discourse about video games. Regarding fans' paratexts as valid voices of video game criticism, one must acknowledge the tactics used by various communities of players (on the Internet discussion boards, as well as YouTube or Twitch.tv themselves) to control and evaluate the feedback and concerns of video game users. While live video forms of games critique, such as podcasts, found their place on the most popular platforms, their impact is noticeably niche compared to the more inclusive forms, such as Reddit or individual streamers' variations of let's play videos. The supported interaction format, interface properties and monetization strategies implemented by Twitch.tv and Douyu.com heavily influence the ways in which broadcasted footage is selected and used. This also pertains to the popularity of fragmented gameplay clips or gifs shared among gaming communities. The paratextual activities demonstrated by fans not only disrupt the play and interview frames of reference, but also establish a very powerful discourse on their own, which can be understood as a form of dialogue with the streamers and game developers alike. Platforms such as Twitch.tv and Douyu.com reflect the proliferating crowdsource trends in the video game industry, with forms of interacting with the product at various stages of its development cycle establishing new practices of talking in and about video games.

\section{Acknowledgments}

I would like to thank the anonymous reviewers for their in-depth constructive criticism and invaluable suggestions. Research for this paper was partially conducted during the work on my PhD thesis (The Concept of Work in AAA Video Games since 1993, unpublished).

\section{References}

Anderson, S.L. (2017). Watching People is Not a Game: Interactive Online Corporeality, Twitch.tv and Videogame Streams. Game Studies, vol. 17, issue 1.

Berglund, A., Magnusson, F., \& Stöckel, F. (2015). Investigating crossmedia branding strategies in online game streaming: A qualitative journey into the hearts and 
minds of todays arising online entertainers. Institutionen för informatic, Umeå University.

Bogost, I. (2009). You Played That? Game Studies Meets Game Criticism. Panel Discussion Paper, DiGRA 2009.

Chun, W.H.K. (2011). Programmed Visions. Software and Memory. MA: MIT Press.

Consalvo, M. (2007). Cheating: Gaining Advantage in Videogames. Cambridge, MA: The MIT Press.

Consalvo, M. (2017). Player one, playing with others virtually: What's next in game and player studies. Critical Studies in Media Communication, 34(1): 84-87.

Deterding, S. (2017). The Pyrrhic Victory of Game Studies. Assessing the Past, Present, and Future of Interdisciplinary Game Research. Games and Culture, vol. 12, issue 6.

Fargo, B. (2017). Interview with the Codex about Torment: Tides of Numenera. Retrieved from http://www.rpgcodex.net/content.php?id=10604

Ford, D. (2016). “eXplore, eXpand, eXploit, eXterminate”: Affective Writing of Postcolonial History and Education in Civilization V. Game Studies, vol. 16, issue 2.

Gros, D., Wanner, B., Hackenholt, A., Zawadzki, P. \& Knautz, K. (2017). World of Streaming. Motivation and Gratification on Twitch. In: Proceedings of Social Computing and Social Media. Human Behavior: 9th International Conference, Part I. Vancouver, Canada, pp. 44-57.

Gandolfi, E. (2016). To watch or to play, it is in the game: The game culture on Twitch. tv among performers, plays and audiences. Journal of Gaming \& Virtual Worlds, 8(1): 63-82.

Ghys, T. (2012). Technology Trees: Freedom and Determinism in Historical Strategy Games. Game Studies, vol. 12, issue 1.

Gray, J. (2010). Show Sold Separately. Promos, Spoilers, and Other Media Paratexts. New York and London: NY University Press.

Hamilton, W.A., Garretson, O., Kerne, A. (2014). Streaming on Twitch: Fostering Participatory Communities of Play within Live Mixed Media. Paper presented during CHI 2014 Conference, Ontario, Canada.

Howarth, D. (2000). Discourse. Philadelphia: Open University Press, Buskingham.

Jenkins, H., Ford, S., \& Green, J. (2013). Spreadable Media: Creating Value and Meaning in a Networked Culture. New York: NY University Press.

Jungar, E. (2016). Streaming Video Games: Copyright Infringement or Protected Speech? Press Start, vol. 3, issue 2. 
Juul, J. (2009). A Casual Revolution. Reinventing Video Games and their Players. Cambridge, Mass.: MIT Press.

Karhulahti, V.-M. (2016). Prank, Troll, Gross and Gore: Performance Issues in Esport Live-Streaming. Proceedings of 1st International Joint Conference of DiGRA and FDG. Available online at: http://www.digra.org/wp-content/uploads/digitallibrary/paper_110.compressed.pdf

Lu, Z., Xia, H., Heo, S., \& Wigdor, D. (2017). You Watch, You Give, and You Engage: A Study of Live Streaming Practices in China. Paper presented during The ACM 2018 CHI Conference on Human Factors in Computing Systems, At Montréal, Canada.

$\mathrm{Mu} \mathrm{Hu}$, Mingli Zhang, Yu Wangon (2017). Why do audiences choose to keep watching on live video streaming platforms? An explanation of dual identification framework. Computers in Human Behavior, vol. 75: 594-606.

Paul, Ch. A. (2011). Optimizing Play: How Theorycraft Changes Gameplay and Design. Game Studies, vol. 11, issue 2.

Recktenwald, D. (2017a). Analizing the Discourse on Twitch. Extended Abstract Presented at Chinese DiGRA 2017.

Recktenwald, D. (2017b). Toward a transcription and analysis of live streaming on Twitch. Journal of Pragmatics, vol. 115: 68-81.

Švelch, J. (2015). Towards a typology of video game trailers: Between the ludic and the cinematic. Game. The Italian Journal of Game Studies, vol. 4.

Zhu, Z., Yang, Z., Dai, Y. (2017), Understanding the Gift-Sending Interaction on LiveStreaming Video Websites. In: Meiselwitz G. (Eds.), Social Computing and Social Media. Human Behavior. SCSM 2017. Lecture Notes in Computer Science, vol. 10282.

Ludography

Firaxis Games (2016). Sid Meier's Civilization VI [PC]. USA: 2k Games.

Simtex (1993). Master of Orion [PC]. USA: MicroProse.

Grinding Gear Games (2013- ). Path of Exile [PC]. New Zeland: Grinding Gear Games.

Abstract

This work concerns online streaming platforms centered on broadcasting video games-related content, such as Twitch.tv and Douyu.com. The aim of this article is two-fold: first, to point out the possible research methods regarding online streaming services as mediums for video game criticism, and second, to assess the potential of 
these platforms for generating critical discourse about video games. The methodology of the research is based on Mia Consalvo's take on paratextual frameworks and Veli-Matti Karhulahti's notions of interview and play frames regarding the activity of video games streaming. In this article, the initial characterization of Twitch.tv and Douyu.com is followed by an analysis on how the work of streamers as content creators can influence the reception of video games. Afterwards, a reading of analyzed phenomena based on paratextual framework is proposed. The last section juxtaposes the initial findings with the perspective of changing viewership figures in relation to selected ludic properties of games. In conclusion, it is stated that the analyzed streaming platforms reflect the proliferating crowdsource trends in the video game industry, with forms of interacting with the product at various stages of its development cycle establishing new practices of talking in and about video games.

Keywords: streaming platforms, paratexts, frames, critical discourse

\section{Author}

Mateusz Felczak (mateuszvf@gmail.com) - PhD, researcher at the Institute of the Middle and Far East, Jagiellonian University. His research interests include cognitive capitalism, e-sports, post-colonial theory, Chinese game market and the mechanisms of modeling the reception and distribution of computer games, especially in the AAA segment. Member of the Game Research Centre in Kraków, Poland. 